\title{
STAT4 Gene Polymorphisms Are Associated with Susceptibility and ANA Status in Primary Biliary Cirrhosis
}

\author{
Satoru Joshita, ${ }^{1}$ Takeji Umemura, ${ }^{1}$ Minoru Nakamura, ${ }^{2}$ \\ Yoshihiko Katsuyama, ${ }^{3}$ Soichiro Shibata, ${ }^{1}$ Takefumi Kimura, ${ }^{1}$ Susumu Morita, ${ }^{1}$ \\ Michiharu Komatsu, ${ }^{1}$ Akihiro Matsumoto, ${ }^{1}$ Kaname Yoshizawa, ${ }^{1}$ Hiromi Ishibashi, ${ }^{2}$ \\ Eiji Tanaka, ${ }^{1}$ and Masao Ota ${ }^{4}$ \\ ${ }^{1}$ Division of Gastroenterology and Hepatology, Department of Medicine, Shinshu University School of Medicine, \\ 3-1-1 Asahi, Matsumoto 390-8621, Japan \\ ${ }^{2}$ Clinical Research Center, NHO Nagasaki Medical Center and Department of Hepatology, \\ Nagasaki University Graduate School of Biomedical Sciences, 2-1001-1 Kubara, Omura 856-8562, Japan \\ ${ }^{3}$ Department of Pharmacy, Shinshu University Hospital, 3-1-1 Asahi, Matsumoto 390-8621, Japan \\ ${ }^{4}$ Department of Legal Medicine, Shinshu University School of Medicine, 3-1-1 Asahi, Matsumoto 390-8621, Japan
}

Correspondence should be addressed to Takeji Umemura; tumemura@shinshu-u.ac.jp and Masao Ota; otamasao@shinshu-u.ac.jp

Received 9 October 2013; Revised 27 November 2013; Accepted 16 December 2013; Published 4 February 2014

Academic Editor: Gad Rennert

Copyright (C) 2014 Satoru Joshita et al. This is an open access article distributed under the Creative Commons Attribution License, which permits unrestricted use, distribution, and reproduction in any medium, provided the original work is properly cited.

Recent genome-wide association studies suggest that genetic factors contribute to primary biliary cirrhosis (PBC) susceptibility. Although several reports have demonstrated that the interleukin (IL) 12 signaling pathway is involved in PBC pathogenesis, its precise genetic factors have not been fully clarified. Here, we performed an association analysis between IL12A, IL12RB, and signal transducer and activator of transcription 4 (STAT4) genetic variations and susceptibility to PBC. Single nucleotide polymorphisms (SNPs) were genotyped in 395 PBC patients and 458 healthy subjects of Japanese ethnicity and evaluated for associations with PBC susceptibility, anti-nuclear antibody (ANA) status, and anti-mitochondrial antibody (AMA) status. We detected significant associations with PBC susceptibility for several STAT4 SNPs (rs10168266; $P=9.4 \times 10^{-3}$, rs11889341; $P=1.2 \times 10^{-3}, \mathrm{rs} 7574865$; $P=4.0 \times 10^{-4}, \mathrm{rs} 8179673 ; P=2.0 \times 10^{-4}$, and rs10181656; $\left.P=4.2 \times 10^{-5}\right)$. Three risk alleles $($ rs $7574865 ; P=0.040, \mathrm{rs} 8179673 ;$ $P=0.032$, and rs10181656; $P=0.031$ ) were associated with ANA status, but not with AMA positivity. Our findings confirm that STAT4 is involved in PBC susceptibility and may play a role in ANA status in the Japanese population.

\section{Introduction}

Primary biliary cirrhosis (PBC) is an autoimmune liver disease characterized by destruction of intrahepatic bile ducts and development of hepatic fibrosis that often progress to cirrhosis and liver failure [1]. The etiology of PBC remains poorly understood and is considered to be complex, [2-4] whereby a combination of inherited genetic predisposition factors and environmental exposure is likely required for disease development. Several genetic characteristics have specifically been implicated in PBC etiology in the Japanese population, including the HLA DRB1 ${ }^{*} 08: 03-D Q B 1^{*} 06: 01$ haplotype and single nucleotide polymorphisms (SNPs) in the cytotoxic T-lymphocyte-associated protein 4 and ataxin 2binding protein 1 genes [5-7].

Recent genome-wide association studies (GWAS) have identified a number of HLA and non-HLA loci with possible relevance to the development of PBC. However, these studies often uncovered different loci in the same signaling pathways across ethnicities; [8-12] genetic variants of the IL12 $\alpha$-chain 
(IL12A) and IL12RB2 genes were associated with disease susceptibility in Caucasian studies, [8-11] but such associations have not been confirmed in the Japanese [12].

Signal transducer and activator of transcription 4 (STAT4) is a transcription factor belonging to the STAT family [13] that is required for the development of Th1 cells from naïve CD4+ T cells [14] and IFN- $\gamma$ production in response to IL12 [15]. Two chains of the IL12 receptor form a heterodimer after IL12 binding and activate the receptor-associated JAK kinases JAK2 and TYK2. STAT4 is phosphorylated by these tyrosine kinases, homodimerizes via its src homology 2 ( $\mathrm{SH} 2$ ) domain, and then translocates into the nucleus to activate cytokine-responsive gene transcription [16]. While early GWAS initially showed a weak association between STAT4 polymorphisms and PBC susceptibility, [8-10] recent investigations have confirmed a definite link between the two $[11,12]$ and have indicated that common pathogenic pathways, such as IL12 signaling, play an essential and nonredundant role in the development of this disease and some of its clinical features.

Anti-mitochondrial antibody (AMA) positivity is the serologic hallmark of PBC. AMA titers tend to be stable over time in individual patients and do not correlate with disease severity or rate of progression $[1,17]$. Antinuclear antibodies (ANA) are found in up to $70 \%$ of patients with $\mathrm{PBC}$ and are suggested to be associated with more rapid disease progression and a poorer prognosis [18]. Positivity for anti-gp210 and anti-centromere antibodies has been related to $\mathrm{PBC}$ progression as well $[19,20]$. Since the association between genetic polymorphisms and autoantibody production has not yet been elucidated, we investigated whether such polymorphisms contributed to a genetic predisposition to $\mathrm{PBC}$ and autoantibody production in the Japanese population.

\section{Patients and Methods}

2.1. Ethics Statement. This study was approved by the ethics committees of both participating institutions (Shinshu University School of Medicine, Matsumoto, Japan, and the National Hospital Organization Nagasaki Medical Center, Omura, Japan), and written informed consent was obtained from all participants. The study was conducted in accordance with the principles of the Declaration of Helsinki.

2.2. Subjects. We analyzed a total of 853 subjects (395 PBC patients and 458 sex-matched healthy controls) enrolled at Shinshu University Hospital, Matsumoto, Japan, and the National Hospital Organization Nagasaki Medical Center, Omura, Japan. As the subjects had no direct relatives of nonJapanese ethnicity, their racial background was considered to be uniformly Japanese. A part of this study's participants had been enrolled in previous genetic association studies [5-7, 12, 21-25]. In particular, 298 of 395 patients (75.4\%) had been included in an earlier GWAS from Japan and were defined as the GWAS cohort in this analysis [12]. The remaining $97(24.6 \%)$ patients were newly diagnosed as having PBC and were defined as the replication cohort. Newly enrolled
TABLE 1: Demographic and clinical data of patients.

\begin{tabular}{lc}
\hline Characteristic & $n=395$ \\
\hline Median age, years (range) & $58(28-87)$ \\
Female/male & $338 / 57$ \\
Autoantibody & \\
AMA-positive, $n(\%)$ & $369(93.4)$ \\
ANA-positive, $n(\%)$ & $271(67.6)$ \\
Cenp positive, $n(\%)$ & $119 / 362(32.9)^{*}$ \\
gp210 positive, $n(\%)$ & $80 / 260(30.8)^{*}$ \\
\hline
\end{tabular}

AMA: anti-mitochondrial antibody; ANA: anti-nuclear antibody; Cenp: anti-centromere antibody; gp210: gp210 antibody.

* Only patients who were assessed for Cenp and gp210 are reported.

control subjects were volunteers from hospital staff who had indicated the absence of any major illnesses in a standard questionnaire and whose racial background was considered to be uniformly Japanese. The sex-matched control group consisted of 384 women and 74 men with no direct familial relations.

The diagnosis of PBC was determined based on criteria from the American Association for the Study of Liver Diseases [26]. Serum AMA, which is specific for the pyruvate dehydrogenase complex-E2 component, was measured by the enzyme-linked immunosorbent assay (ELISA). An index of greater than 7 was considered to be a positive result. Serum ANA was determined by immunofluorescence using HEp-2 cells, whereby a titer of $\geq 40$ was considered to be positive, as reported previously [27]. Patterns of ANA reactivity were recorded as well. Serum anti-centromere antibody was detected using commercially available ELISA kits (MBL, Nagoya, Japan), for which an index of $>5 \mathrm{U} / \mathrm{mL}$ was considered to be positive. Serum gp-210 antibody was also measured by ELISA, whereby an index of $>10 \mathrm{U} / \mathrm{mL}$ was considered to be a positive result, as previously described [19]. All patients were negative for hepatitis B surface antigen and antibodies to the hepatitis $\mathrm{C}$ and human immunodeficiency viruses.

\subsection{IL-12 Signaling-Related SNP Genotyping. Genomic DNA} from patients was isolated by phenolic extraction of sodium dodecyl sulfate-lyzed and proteinase K-treated cells, as described previously [28], and adjusted to a concentration of $10-15 \mathrm{ng} / \mu \mathrm{L}$.

IL12A (rs574808), IL12RB (rs3790567), and STAT4 (rs7574865) SNPs were selected based on reported PBC susceptibility [8-12]. Since the STAT4 (rs7574865) SNP was found to be significantly associated with PBC, we genotyped an additional 7 SNPs located in this gene (rs10168266, rs7594501, rs16833239, rs11889341, rs8179673, rs10181656, and rs6752770) that were not evaluated in the earlier Japanese GWAS using an SNP Genotyping Kit (Applied Biosystems, Tokyo, Japan). These SNPs were selected based on previous reports [8-12, 29-31]. Polymerase chain reaction (PCR) was performed with TaqMan Assays (7500 Real Time PCR System; Applied Biosystems, Foster City, California, USA) following the manufacturer's instructions. 
TABLE 2: IL12A, IL12RB, and STAT4 SNPs in PBC patients and healthy subjects.

\begin{tabular}{|c|c|c|c|c|c|c|c|c|c|}
\hline Gene & $\mathrm{db}$ SNP & $\begin{array}{c}\text { Allele } \\
\text { minor/major }\end{array}$ & $\begin{array}{l}\text { GWAS cohort } \\
\text { patients }(\%) \\
(n=302)\end{array}$ & $\begin{array}{c}\text { Replication cohort } \\
\text { patients (\%) } \\
(n=93)\end{array}$ & $\begin{array}{c}\text { Combined cohort } \\
\text { patients }(\%) \\
(n=395)\end{array}$ & $\begin{array}{c}\text { Controls }(\%) \\
(n=458)\end{array}$ & $P$ & OR & $95 \%$ CI \\
\hline IL12A & rs574808 & $\mathrm{C} / \mathrm{T}$ & 17.2 & 18.3 & 17.5 & 18.1 & 0.75 & & \\
\hline IL12RB & rs3790567 & $\mathrm{A} / \mathrm{G}$ & 26.8 & 22.0 & 25.7 & 22.9 & 0.18 & & \\
\hline STAT4 & rs7574865 & $\mathrm{T} / \mathrm{G}$ & 40.6 & 46.8 & 41.9 & 33.5 & $4.0 \times 10^{-4}$ & 1.43 & $1.17-1.74$ \\
\hline
\end{tabular}

IL12: interleukin 12; STAT4: signal transducer activator transcription 4; PBC: primary biliary cirrhosis; SNP: single nucleotide polymorphism; OR: odds ratio; CI: confidence interval.

TABLE 3: STAT4 SNPs in PBC patients and healthy subjects.

\begin{tabular}{|c|c|c|c|c|c|c|c|}
\hline $\mathrm{db}$ SNP & Allele minor/major & Patients $(\%)(n=395)$ & Controls $(\%)(n=458)$ & $P$ & $P_{c}$ & OR & $95 \% \mathrm{CI}$ \\
\hline rs10168266 & $\mathrm{A} / \mathrm{G}$ & 34.5 & 28.7 & $9.4 \times 10^{-3}$ & 0.038 & 1.31 & $1.07-1.61$ \\
\hline rs7594501 & $\mathrm{A} / \mathrm{G}$ & 12.7 & 16.7 & 0.021 & & & \\
\hline rs16833239 & $\mathrm{T} / \mathrm{C}$ & 13.0 & 16.4 & 0.046 & & & \\
\hline rs11889341 & $\mathrm{T} / \mathrm{C}$ & 39.1 & 31.7 & $1.2 \times 10^{-3}$ & $9.6 \times 10^{-3}$ & 1.39 & $1.14-1.69$ \\
\hline rs7574865 & $\mathrm{T} / \mathrm{G}$ & 41.9 & 33.5 & $4.0 \times 10^{-4}$ & $3.2 \times 10^{-3}$ & 1.43 & $1.17-1.74$ \\
\hline rs8179673 & $\mathrm{G} / \mathrm{A}$ & 42.1 & 33.5 & $2.0 \times 10^{-4}$ & $1.6 \times 10^{-3}$ & 1.44 & $1.19-1.75$ \\
\hline rs10181656 & $\mathrm{G} / \mathrm{C}$ & 43.3 & 33.7 & $4.2 \times 10^{-5}$ & $3.4 \times 10^{-4}$ & 1.50 & $1.24-1.83$ \\
\hline rs6752770 & $\mathrm{G} / \mathrm{A}$ & 15.0 & 17.8 & 0.120 & & & \\
\hline
\end{tabular}

STAT4: signal transducer activator transcription 4; PBC: primary biliary cirrhosis; SNP: single nucleotide polymorphism; OR: odds ratio; $P_{c}$ : corrected $P$ value; CI: confidence interval.

2.4. Statistical Analysis. All examined SNPs in control groups were in the Hardy-Weinberg equilibrium. The R-software "Haploview" [32] version 4.2 was used to evaluate the haplotype structure of the 8 STAT4 SNPs. Pairwise linkage disequilibrium (LD) patterns and haplotype frequency analysis for all SNPs in patients and controls were assessed by the block definition established by Gabriel et al. [33]. We assessed the significance of allele distribution between patients and controls using the $\chi^{2}$ test by means of $2 \times 2$ comparisons. A $P$ value of less than 0.05 was considered to be statistically significant. We adjusted $P$ values using Bonferroni's correction by multiplying each locus by $8\left(P_{c}\right)$. Association strength was estimated by calculating the odds ratio (OR) and 95\% confidence interval (CI). Statistical analysis of data was performed using SPSS 21.0 software (IBM, Armonk, New York).

\section{Results}

3.1. Genotyping of IL12 Signaling-Related SNPs. To clarify the genetic susceptibility to PBC based on previously reported IL12 signaling, a total of 395 Japanese patients with PBC and 458 healthy Japanese controls were enrolled for an association analysis of IL12A (rs574808), IL12RB (rs3790567), and STAT4 (rs7574865) SNPs (Table 2). Whereas the IL12A and IL12RB SNPs were not associated with PBC, the rs7574865 SNP in STAT4 showed a positive association with $\mathrm{PBC}$ susceptibility (41.9\% versus $33.5 \%$; $P=4.0 \times 10^{-4}, \mathrm{OR}=1.43,95 \% \mathrm{CI}=$ 1.17-1.74). To further examine its role in PBC, we selected an additional 7 SNPs from STAT4 (rs10168266, rs7594501, rs16833239, rs11889341, rs8179673, rs10181656, and rs6752770) and genotyped them in all patients and controls. The minor allele frequencies of $\mathrm{A}$ at rs10168266, $\mathrm{T}$ at rs11889341, T at rs7574865, G at rs8179673, and G at rs10181656 were significantly increased in $\mathrm{PBC}$ patients as compared with controls $\left(P=9.4 \times 10^{-3}, P=1.2 \times 10^{-3}, P=4.0 \times 10^{-4}\right.$, $P=2.0 \times 10^{-4}$, and $P=4.0 \times 10^{-5}$, resp.) (Table 3 ).

3.2. Distribution of STAT4 Haplotypes among PBC Patients and Controls. We firstly defined LD blocks for the 8 SNPs in STAT4 (Figure 1). The STAT4 region was divided into two haplotype blocks, with substantial LD among the SNPs in each block. To estimate haplotype frequency and analyze haplotype association with PBC, we selected tag SNPs using the Tagger algorithm from the Haploview program. Three tag SNPs (rs7594501, rs16833239, and rs11889341) in block A and 3 tag SNPs (rs7574865, rs8179673, and rs10181656) in block $\mathrm{B}$ were captured from pairwise measures of LD. The top 3 haplotype frequencies in both blocks are shown in Table 4. Haplotype 2 (GCT) in block A was significantly associated with PBC susceptibility (40.3\% versus $32.2 \%$; $P=3.5 \times 10^{-3}$, OR 1.43, 95\% CI 1.12-1.81), as was haplotype 5 (TGG) in block B (43.3\% versus $33.6 \%$; $P=6.0 \times 10^{-4}$, OR $1.51,95 \%$ CI $1.19-$ 1.91). In contrast, protective effects were seen for haplotype 4 (GAC) in block B (53.9\% versus $65.4 \% ; P=5.0 \times 10^{-5}$, OR $0.62,95 \%$ CI $0.49-0.78)$.

3.3. Associations between STAT4 SNPs, Haplotypes, and Autoantibodies. The PBC patients enrolled in this study were highly positive for disease-specific autoantibodies (Table 1). There were no significant differences with regard to ANA positivity between 250 of 369 AMA-positive patients $(67.8 \%)$ and 21 of 26 AMA-negative patients $(80.8 \%)(P=0.245)$. Among the 8 STAT4 SNPs, the frequencies of 3 minor alleles 
TABLE 4: STAT4 haplotypes in PBC patients and healthy subjects.

\begin{tabular}{|c|c|c|c|c|c|c|c|c|c|}
\hline Block & Haplotype & & SNPs & & $\begin{array}{c}\text { Patients (\%) } \\
\left(n^{*}=790\right)\end{array}$ & $\begin{array}{c}\text { Controls }(\%) \\
\left(n^{*}=916\right)\end{array}$ & $P$ & OR & $95 \% \mathrm{CI}$ \\
\hline \multirow[t]{4}{*}{ A } & & rs7594501 & rs16833239 & rs11889341 & & & & & \\
\hline & 1 & G & C & C & 46.4 & 50.0 & 0.222 & & \\
\hline & 2 & G & $\mathrm{C}$ & $\mathrm{T}$ & 40.3 & 32.2 & $3.5 \times 10^{-3}$ & 1.43 & $1.12-1.81$ \\
\hline & 3 & $\mathrm{~A}$ & $\mathrm{~T}$ & $\mathrm{C}$ & 11.9 & 15.9 & 0.045 & & \\
\hline \multirow[t]{4}{*}{ B } & & rs7574865 & rs8179673 & rs10181656 & & & & & \\
\hline & 4 & G & A & C & 53.9 & 65.4 & $5.0 \times 10^{-5}$ & 0.62 & $0.49-0.78$ \\
\hline & 5 & $\mathrm{~T}$ & G & G & 43.3 & 33.6 & $6.0 \times 10^{-4}$ & 1.51 & $1.19-1.91$ \\
\hline & 6 & G & A & G & 2.1 & 0.0 & $3.0 \times 10^{-4}$ & & \\
\hline
\end{tabular}

STAT4: signal transducer activator transcription 4; PBC: primary biliary cirrhosis; SNP: single nucleotide polymorphism; OR: odds ratio; CI: confidence interval; $n^{*}$ : values for $n^{*}$ indicate two times the number of individuals since each person carries two haplotypes.
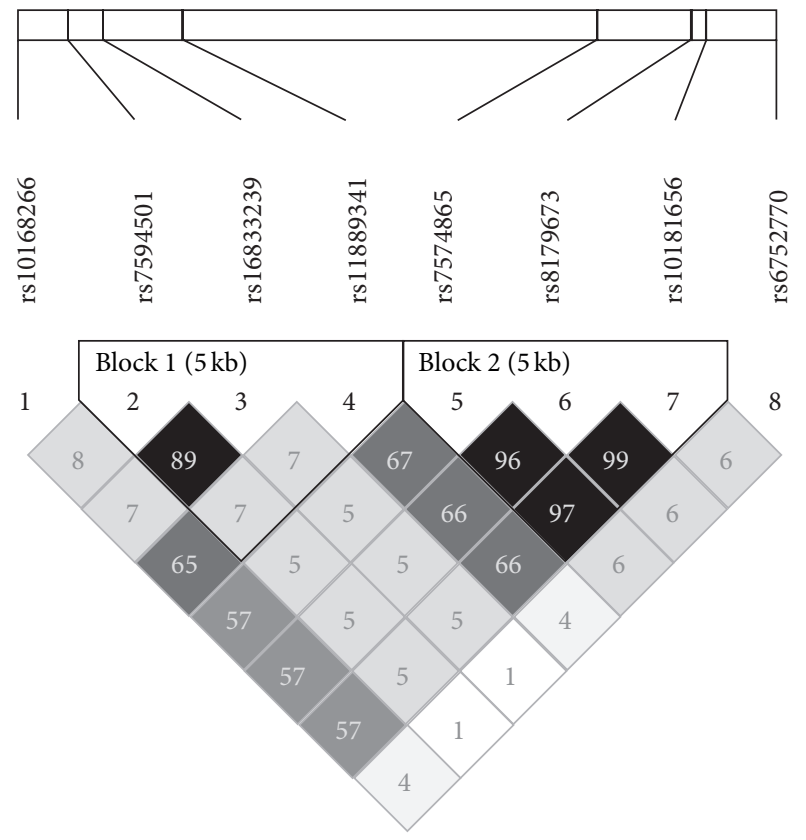

FIGURE 1: Linkage disequilibrium (LD) plot of 8 SNPs in STAT4 in 458 healthy subjects. Values of $r^{2}$ corresponding to each SNP pair are expressed as a percentage and shown within the respective square.

( $\mathrm{T}$ at rs7574865, G at rs8179673, and $\mathrm{G}$ at rs10181656) were increased in ANA-positive PBC patients as compared with ANA-negative patients $(P=0.040, P=0.032$, and $P=0.031$, resp.) (Table 5), but these statistical significances disappeared after correction. Haplotype 5 (TGG) in block B was significantly correlated with ANA (44.3\% versus $36.3 \% ; P=0.035$, OR 1.40, 95\% CI 1.02-1.90) (Table 6). Interestingly, haplotype 5 was also significantly associated with the speckled pattern of ANA as compared with the nonspeckled pattern $(45.8 \%$ versus $36.3 \% ; P=0.029$, OR $1.48,95 \%$ CI $1.04-2.12$ ) (data not shown). No STAT4 SNPs or haplotypes were associated with other autoantibody positivity or ANA pattern, such as discrete speckled pattern, homogenous pattern, nucleolar pattern, or peripheral pattern.

\section{Discussion}

In the present study, we investigated the association between STAT4 SNPs and PBC susceptibility and its clinical significance in the Japanese population. Our key findings were as follows: (1) specific STAT4 polymorphisms and haplotypes were significantly associated with PBC susceptibility or protection; (2) there were no significant genetic associations between IL12A and IL12RB SNPs and PBC susceptibility, in contrast to studies of Caucasians; [8-11] and (3) there was a moderate relationship between STAT4 SNPs and ANApositive, but not AMA-positive, PBC patients.

STAT4 lies in the signaling pathways of several cytokines, such as IL12, type I interferon, and IL23. This time, a newly diagnosed PBC cohort, albeit small, replicated the previous finding by Japanese GWAS [12] that a STAT4 SNP at rs7574865 was associated with susceptibility to PBC. To evaluate the power of this study, larger association studies of other ethnicities, including Chinese and Caucasian populations, are required because small sample sizes may lead to false positive or negative results. Moreover, we identified 4 additional SNPs in STAT4 that conferred susceptibility to $\mathrm{PBC}$ and were consistent with findings of GWAS of other ethnicities [11]. Haplotype analysis showed that 3 of the identified risk SNPs, rs7574865, rs8179673, and rs10181656, were located in the same LD block (Figure 1 and Table 4). These SNPs and this haplotype have been linked with several autoimmune diseases, including rheumatoid arthritis and systemic lupus erythematosus (SLE) [29-31, 34-38]. In particular, it has been reported that SNPs at rs7574865 are associated with numerous other autoimmune diseases [35-38]. As we focused only on STAT4 polymorphisms, we must concede that our study is rather limited in depth and scope compared with recent immunogenic studies $[39,40]$ using the Immunochip. However, our findings support the notion that SNPs and haplotypes in STAT4 may contribute to the development of $\mathrm{PBC}$ and other autoimmune diseases.

In addition to our own, two earlier studies from Japan [12, 41] showed that IL12A and IL12RB SNPs were not associated with $\mathrm{PBC}$, which was in contrast to strong associations found in Caucasian population studies [8-10, 42]. Similarly, GWAS of the Japanese have identified novel significant susceptibility 


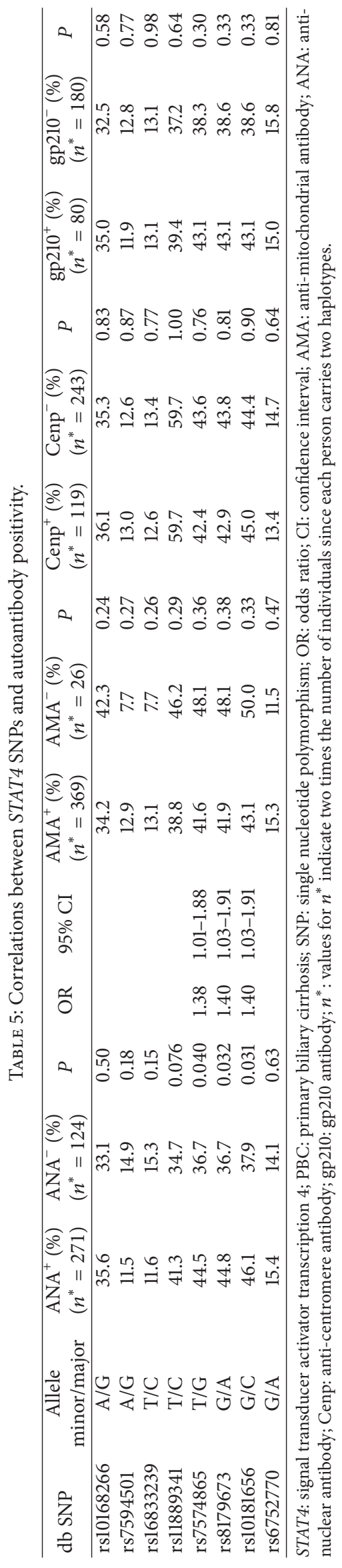




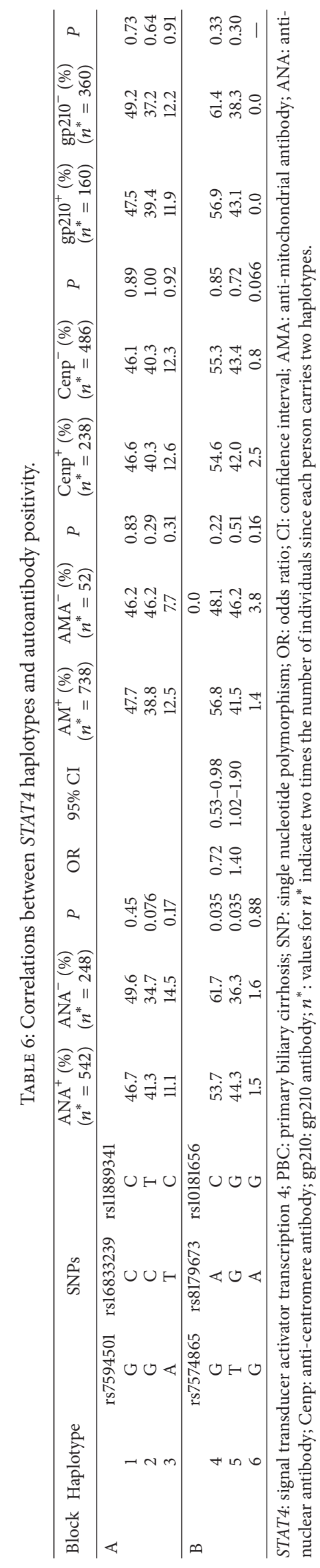


loci for PBC, such as TNFSF15 and POU2AF1, which have not been identified in GWAS of populations of European descent. Meanwhile, Peters et al. found that liver damage severity at clinical presentation is higher among non-Caucasians than Caucasians for PBC [43]. Hence, although the IL12 pathway through STAT4 plays an essential role in PBC etiology, there is evidence of ethnic differences in genetic susceptibility loci. PBC is also concurrent with other autoimmune diseases, including Sjögren syndrome, [44] rheumatoid arthritis, [45] and cutaneous scleroderma, [46] so we cannot exclude the possibility of genetic overlap among these disorders.

Interestingly, our study showed a moderate association between 3 STAT4 polymorphisms and 2 haplotypes with ANA-positive PBC that was not seen for AMA (Tables 5 and 6). To understand its clinical relevance, we analyzed this association with regard to ANA pattern and found a significant relationship between these SNPs and the speckled pattern of ANA, which was in agreement with a recent metaanalysis showing that the presence of anti-ds-DNA antibodies was associated with rs7574865 within STAT4 polymorphisms in SLE patients. Our results did not support a relationship between STAT4 SNPs and gp210 or anti-centromere antibodies, despite these antibodies having been associated with disease progression and prognosis in PBC patients [19]. Taken together, our data implied that STAT4 SNPs imparted susceptibility to ANA-positive $\mathrm{PBC}$, but for reasons that are still unknown. The mechanisms by which genetic variants are correlated with ANA positivity may be diverse and require further study.

\section{Conclusions}

Our findings confirm that STAT4 SNPs and haplotypes contribute to $\mathrm{PBC}$ susceptibility and may play a role in mediating ANA status. STAT4 appears to factor strongly in the pathogenesis of this and other autoimmune diseases and requires continued study.

\section{Abbreviations}

PBC: Primary biliary cirrhosis

SNP: $\quad$ Single nucleotide polymorphism

GWAS: Genome-wide association study

IL12: Interleukin12

STAT4: Signal transducer and activator of transcription 4

AMA: Anti-mitochondrial antibody

ANA: Anti-nuclear antibody

PBMCs: Peripheral blood mononuclear cells

LD: $\quad$ Linkage disequilibrium

$P_{c}$ : $\quad$ Corrected $P$

OR: Odds ratio

CI: Confidence interval.

\section{Conflict of Interests}

The authors declare that there is no conflict of interests regarding the publication of this paper.

\section{Acknowledgments}

The authors thank Yuki Akahane, Asami Yamazaki, and Toyo Amaki for their technical assistance and Trevor Ralph for his English editorial assistance.

\section{References}

[1] M. M. Kaplan and M. E. Gershwin, "Primary biliary cirrhosis," New England Journal of Medicine, vol. 353, no. 12, pp. 1261-1273, 2005.

[2] C. Selmi, M. J. Mayo, N. Bach et al., "Primary biliary cirrhosis in monozygotic and dizygotic twins: genetics, epigenetics, and environment," Gastroenterology, vol. 127, no. 2, pp. 485-492, 2004.

[3] P. Invernizzi, C. Selmi, I. R. Mackay, M. Podda, and M. E. Gershwin, "From bases to basis: linking genetics to causation in primary biliary cirrhosis," Clinical Gastroenterology and Hepatology, vol. 3, no. 5, pp. 401-410, 2005.

[4] G. M. Hirschfield and P. Invernizzi, "Progress in the genetics of primary biliary cirrhosis," Seminars in Liver Disease, vol. 31, no. 2, pp. 147-156, 2011.

[5] T. Umemura, S. Joshita, T. Ichijo et al., "Human leukocyte antigen class II molecules confer both susceptibility and progression in Japanese patients with primary biliary cirrhosis," Hepatology, vol. 55, no. 2, pp. 506-511, 2012.

[6] S. Joshita, T. Umemura, K. Yoshizawa, Y. Katsuyama, E. Tanaka, and M. Ota, "A2BP1 as a novel susceptible gene for primary biliary cirrhosis in Japanese patients," Human Immunology, vol. 71, no. 5, pp. 520-524, 2010.

[7] S. Joshita, T. Umemura, K. Yoshizawa et al., "Association analysis of cytotoxic T-lymphocyte antigen 4 gene polymorphisms with primary biliary cirrhosis in Japanese patients," Journal of Hepatology, vol. 53, no. 3, pp. 537-541, 2010.

[8] G. M. Hirschfield, X. Liu, C. Xu et al., "Primary biliary cirrhosis associated with HLA, IL12A, and IL12RB2 variants," New England Journal of Medicine, vol. 360, pp. 2544-2555, 2009.

[9] G. M. Hirschfield, X. Liu, Y. Han et al., "Variants at IRF5TNPO3, 17q12-21 and MMEL1 are associated with primary biliary cirrhosis," Nature Genetics, vol. 42, no. 8, pp. 655-657, 2010.

[10] X. Liu, P. Invernizzi, Y. Lu et al., "Genome-wide meta-analyses identify three loci associated with primary biliary cirrhosis," Nature Genetics, vol. 42, pp. 658-660, 2010.

[11] G. F. Mells, J. A. B. Floyd, K. I. Morley et al., "Genomewide association study identifies 12 new susceptibility loci for primary biliary cirrhosis," Nature Genetics, vol. 43, pp. 329-332, 2011.

[12] M. Nakamura, N. Nishida, M. Kawashima et al., "Genomewide association study identifies TNFSF15 and POU2AF1 as susceptibility loci for primary biliary cirrhosis in the Japanese population," American Journal of Human Genetics, vol. 91, pp. 721-728, 2012.

[13] K. Yamamoto, F. W. Quelle, W. E. Thierfelder et al., "Stat4, a novel gamma interferon activation site-binding protein expressed in early myeloid differentiation," Molecular and Cellular Biology, vol. 14, no. 7, pp. 4342-4349, 1994.

[14] M. H. Kaplan, "STAT4: a critical regulator of inflammation in vivo," Immunologic Research, vol. 31, no. 3, pp. 231-242, 2005.

[15] C. M. Bacon, E. F. Petricoin III, J. R. Ortaldo et al., "Interleukin 12 induces tyrosine phosphorylation and activation of STAT4 
in human lymphocytes," Proceedings of the National Academy of Sciences of the United States of America, vol. 92, no. 16, pp. 73077311, 1995.

[16] A. L. Wurster, T. Tanaka, and M. J. Grusby, “The biology of Stat4 and Stat6," Oncogene, vol. 19, no. 21, pp. 2577-2584, 2000.

[17] M. D. van Norstrand, M. Malinchoc, K. D. Lindor et al., "Quantitative measurement of autoantibodies to recombinant mitochondrial antigens in patients with primary biliary cirrhosis: relationship of levels of autoantibodies to disease progression," Hepatology, vol. 25, no. 1, pp. 6-11, 1997.

[18] P. Muratori, L. Muratori, R. Ferrari et al., "Characterization and clinical impact of antinuclear antibodies in primary biliary cirrhosis," American Journal of Gastroenterology, vol. 98, no. 2, pp. 431-437, 2003.

[19] M. Nakamura, H. Kondo, T. Mori et al., "Anti-gp210 and anti-centromere antibodies are different risk factors for the progression of primary biliary cirrhosis," Hepatology, vol. 45, no. 1, pp. 118-127, 2007.

[20] M. Nakamura, Y. Shimizu-Yoshida, Y. Takii et al., "Antibody titer to gp210-C terminal peptide as a clinical parameter for monitoring primary biliary cirrhosis," Journal of Hepatology, vol. 42, no. 3, pp. 386-392, 2005.

[21] Y. Aiba, M. Nakamura, S. Joshita et al., "Genetic polymorphisms in CTLA4 and SLC4A2 are differentially associated with the pathogenesis of primary biliary cirrhosis in Japanese patients," Journal of Gastroenterology, vol. 46, no. 10, pp. 1203-1212, 2011.

[22] S. Morita, S. Joshita, T. Umemura et al., "Association analysis of toll-like receptor 4 polymorphisms in Japanese primary biliary cirrhosis," Human Immunology, vol. 74, no. 2, pp. 219-222, 2013.

[23] T. Inamine, M. Nakamura, A. Kawauchi et al., "A polymorphism in the integrin alphaV subunit gene affects the progression of primary biliary cirrhosis in Japanese patients," Journal of Gastroenterology, vol. 46, no. 5, pp. 676-686, 2011.

[24] Y. Ohishi, M. Nakamuta, N. Ishikawa et al., "Genetic polymorphisms of OCT-1 confer susceptibility to severe progression of primary biliary cirrhosis in Japanese patients," Journal of Gastroenterology, 2013.

[25] T. Inamine, S. Higa, F. Noguchi et al., "Association of genes involved in bile acid synthesis with the progression of primary biliary cirrhosis in Japanese patients," Journal of Gastroenterology, vol. 48, no. 10, pp. 1160-1170, 2013.

[26] K. D. Lindor, M. E. Gershwin, R. Poupon, M. Kaplan, N. V. Bergasa, and E. J. Heathcote, "Primary biliary cirrhosis," Hepatology, vol. 50, no. 1, pp. 291-308, 2009.

[27] T. Umemura, Y. Zen, H. Hamano, S. Kawa, Y. Nakanuma, and K. Kiyosawa, "Immunoglobin G4-hepatopathy: association of immunoglobin G4-bearing plasma cells in liver with autoimmune pancreatitis," Hepatology, vol. 46, no. 2, pp. 463-471, 2007.

[28] M. Ota, T. Seki, N. Nomura et al., "Modified PCR-RFLP method for HLA-DPB1 and -DQA1 genotyping," Tissue Antigens, vol. 38, no. 2, pp. 60-71, 1991.

[29] E. F. Remmers, R. M. Plenge, A. T. Lee et al., "STAT4 and the risk of rheumatoid arthritis and systemic lupus erythematosus," New England Journal of Medicine, vol. 357, no. 10, pp. 977-986, 2007.

[30] S. Kobayashi, K. Ikari, H. Kaneko et al., "Association of STAT4 with susceptibility to rheumatoid arthritis and systemic lupus erythematosus in the Japanese population," Arthritis and Rheumatism, vol. 58, no. 7, pp. 1940-1946, 2008.

[31] S. Sigurdsson, G. Nordmark, S. Garnier et al., "A risk haplotype of STAT4 for systemic lupus erythematosus is over-expressed, correlates with anti-dsDNA and shows additive effects with two risk alleles of IRF5," Human Molecular Genetics, vol. 17, no. 18, pp. 2868-2876, 2008.

[32] J. C. Barrett, B. Fry, J. Maller, and M. J. Daly, "Haploview: analysis and visualization of LD and haplotype maps," Bioinformatics, vol. 21, no. 2, pp. 263-265, 2005.

[33] S. B. Gabriel, S. F. Schaffner, H. Nguyen et al., "The structure of haplotype blocks in the human genome," Science, vol. 296, no. 5576, pp. 2225-2229, 2002.

[34] K. E. Taylor, S. A. Chung, R. R. Graham et al., "Risk alleles for systemic lupus erythematosus in a large case-control collection and associations with clinical subphenotypes," PLoS Genetics, vol. 7, no. 2, Article ID e1001311, 2011.

[35] E. Alonso-Perez, M. Suarez-Gestal, M. Calaza et al., "Further evidence of subphenotype association with systemic lupus erythematosus susceptibility loci: a European cases only study," PloS ONE, vol. 7, no. 9, Article ID e45356, 2012.

[36] A. Lamana, A. Balsa, B. Rueda et al., "The TT genotype of the STAT4 rs7574865 polymorphism is associated with high disease activity and disability in patients with early arthritis," PloS ONE, vol. 7, no. 8, Article ID e43661, 2012.

[37] L. Shen, R. Liu, H. Zhang, Y. Huang, R. Sun, and P. Tang, "Replication study of STAT4 rs7574865 G/T polymorphism and risk of rheumatoid arthritis in a Chinese population," Gene, vol. 526, no. 2, pp. 259-264, 2013.

[38] G. Tong, X. Zhang, W. Tong, and Y. Liu, "Association between polymorphism in STAT4 gene and risk of rheumatoid arthritis: a meta-analysis," Human Immunology, vol. 74, no. 5, pp. 586592, 2013.

[39] J. Z. Liu, M. A. Almarri, D. J. Gaffney et al., "Dense finemapping study identifies new susceptibility loci for primary biliary cirrhosis," Nature Genetics, vol. 44, pp. 1137-1141, 2012.

[40] B. D. Juran, G. M. Hirschfield, P. Invernizzi et al., "Immunochip analyses identify a novel risk locus for primary biliary cirrhosis at 13q14, multiple independent associations at four established risk loci and epistasis between 1p31 and 7q32 risk variants," Human Molecular Genetics, vol. 21, pp. 5209-5221, 2012.

[41] A. Tanaka, P. Invernizzi, H. Ohira et al., "Replicated association of 17q12-21 with susceptibility of primary biliary cirrhosis in a Japanese cohort," Tissue Antigens, vol. 78, no. 1, pp. 65-68, 2011.

[42] G. F. Mells, J. A. B. Floyd, K. I. Morley et al., "Genomewide association study identifies 12 new susceptibility loci for primary biliary cirrhosis," Nature Genetics, vol. 43, no. 11, p. 1164, 2011.

[43] M. G. Peters, A. M. Di Bisceglie, K. V. Kowdley et al., "Differences between Caucasian, African American, and Hispanic patients with primary biliary cirrhosis in the United States," Hepatology, vol. 46, no. 3, pp. 769-775, 2007.

[44] B. Liu, F. C. Zhang, Z. L. Zhang, W. Zhang, and L. X. Gao, "Interstitial lung disease and Sjögren's syndrome in primary biliary cirrhosis: a causal or casual association?" Clinical Rheumatology, vol. 27, no. 10, pp. 1299-1306, 2008.

[45] C. Caramella, J. Avouac, P. Sogni, X. Puéchal, A. Kahan, and Y. Allanore, "Association between rheumatoid arthritis and primary biliary cirrhosis," Joint Bone Spine, vol. 74, no. 3, pp. 279-281, 2007.

[46] M. J. Mayo, R. N. Jenkins, B. Combes, and P. E. Lipsky, "Association of clonally expanded T cells with the syndrome of primary biliary cirrhosis and limited scleroderma," Hepatology, vol. 29, no. 6, pp. 1635-1642, 1999. 


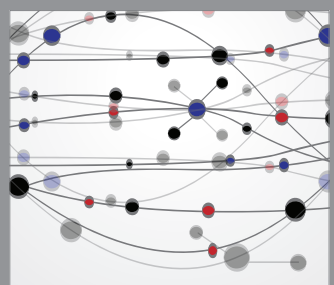

The Scientific World Journal
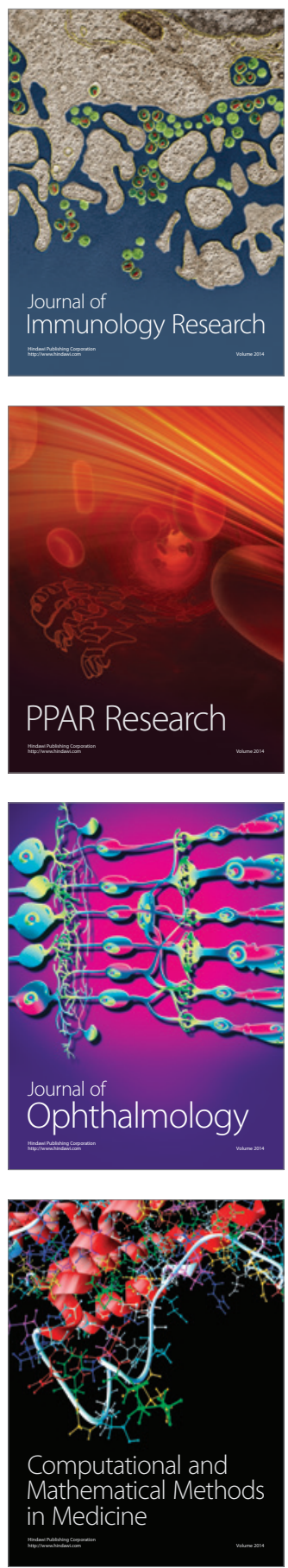

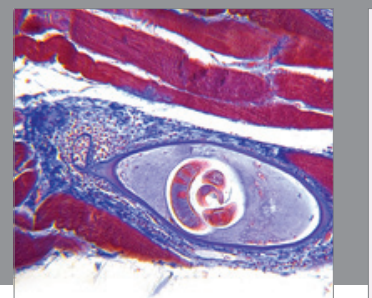

Gastroenterology

Research and Practice
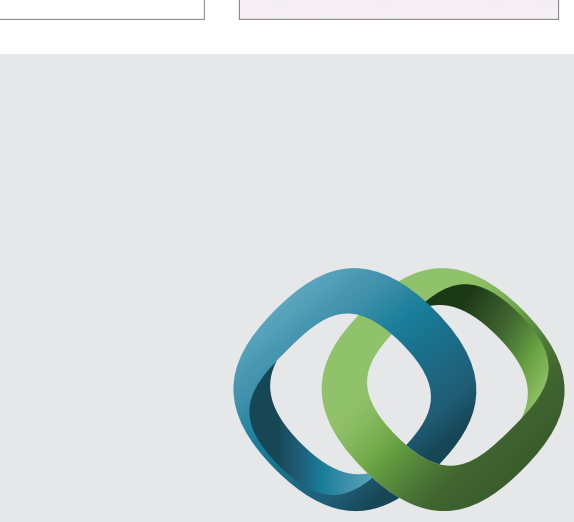

\section{Hindawi}

Submit your manuscripts at

http://www.hindawi.com
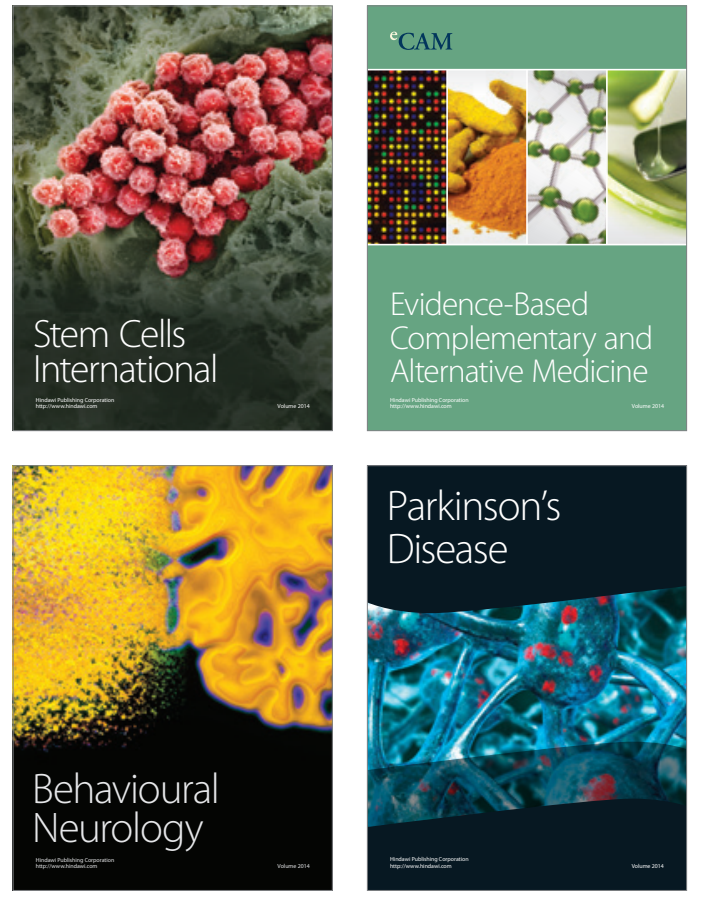
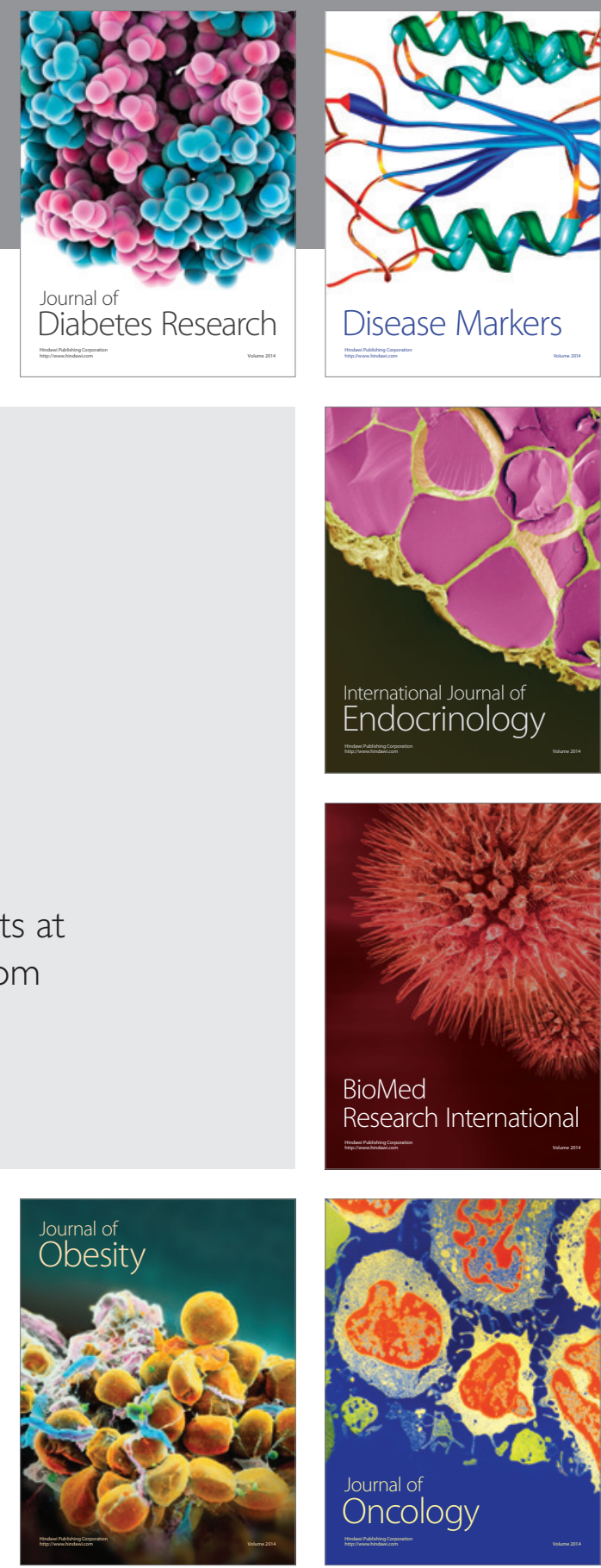

Disease Markers
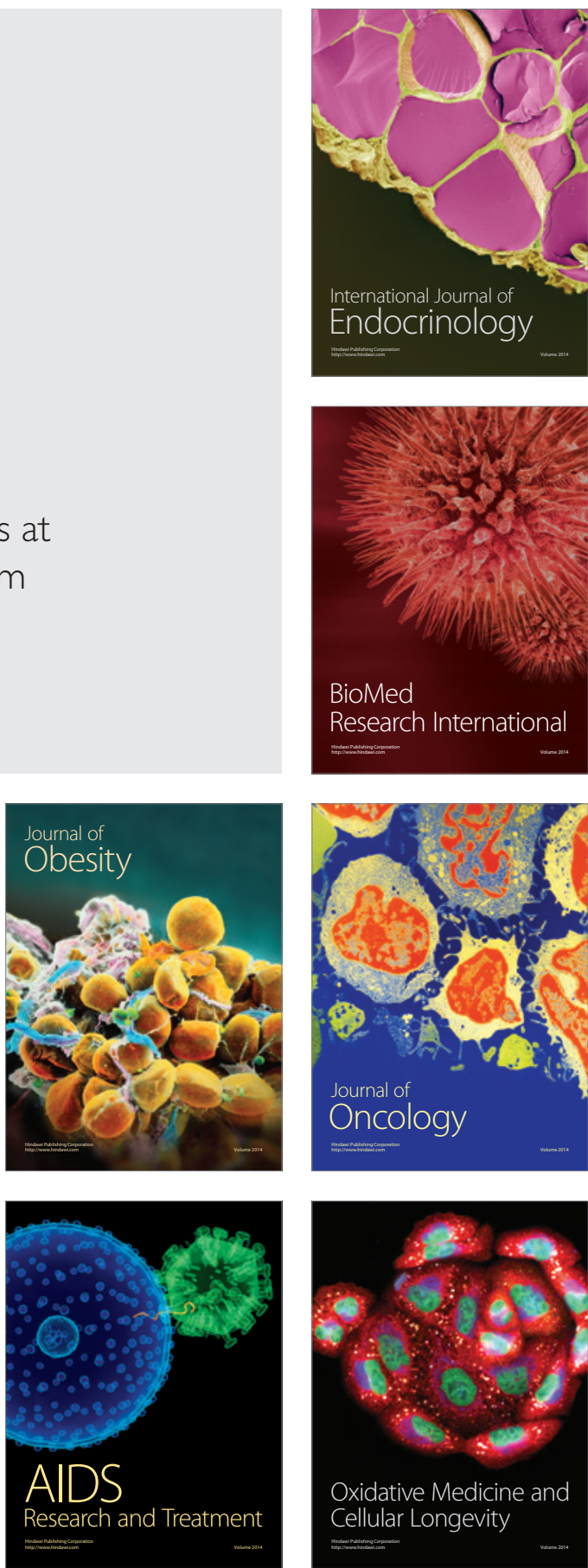